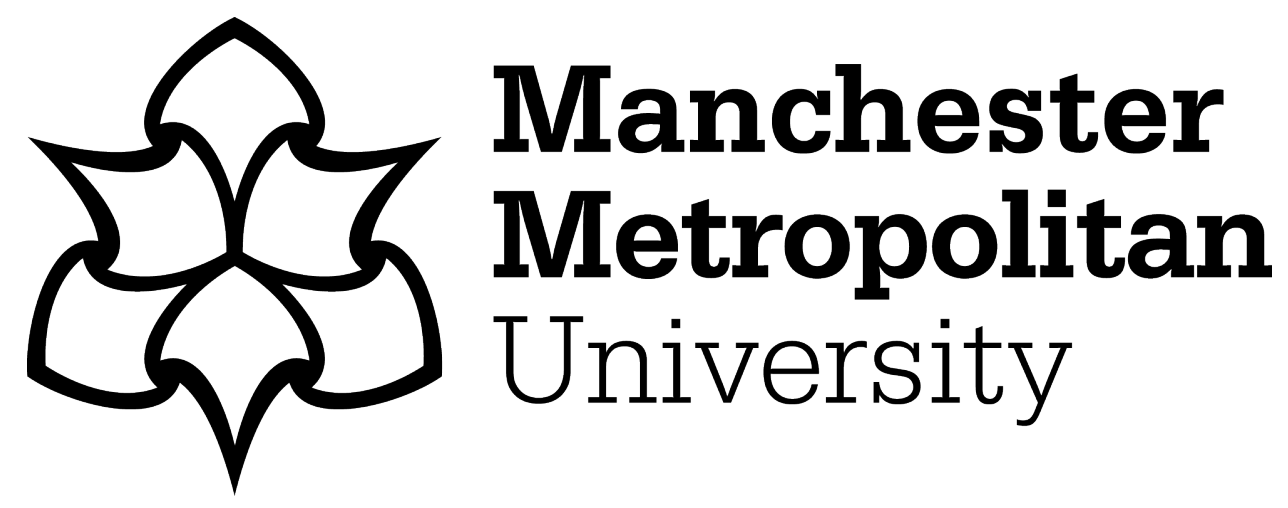

Wingrave, Adele and Jarvis, HL ORCID logoORCID: https://orcid.org/00000001-7825-3671 (2018) The importance of the rehabilitation program following an internal hemipelvectomy and reconstrucion with limb salvage - gait analysis and outcomes: a case study. Disability and Rehabilitation, 41 (17). pp. 2066-2070. ISSN 0963-8288

Downloaded from: https://e-space.mmu.ac.uk/620879/

Version: Accepted Version

Publisher: Taylor \& Francis

DOI: https://doi.org/10.1080/09638288.2018.1457090

Please cite the published version 


\title{
The importance of the rehabilitation program following an internal hemipelvectomy and reconstruction with limb salvage - gait analysis and outcomes: a case study
}

\author{
Adele Wingrave $^{\mathrm{a}}$ and Hannah Jarvis ${ }^{\mathrm{b}}$

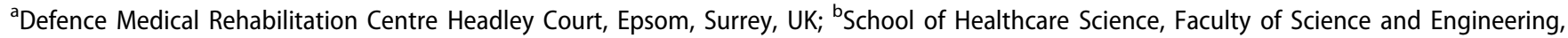 \\ John Dalton Building, Manchester Metropolitan University, Manchester, UK
}

\begin{abstract}
Background: Chondrosarcomas account for approximately $20 \%$ of bone sarcomas, with the most common site being the lower limb. Hemipelvectomies that involve hindquarter amputation have previously been the treatment of choice for pelvic tumors. However, with advances in chemotherapy, radiotherapy, imaging and surgical techniques more patients are now being treated with limb salvage surgery. The rehabilitation outcomes following an internal hemipelvectomy have not been well identified and there is currently little, if any, gait analysis data on rehabilitation following hemipelvectomy in limb salvage patients.

Case description: A 53-year-old male underwent an internal hemipelvectomy with limb salvage followed by a 2-year rehabilitation program. The aim of this case study is to describe temporal and spatial parameters, metabolic energy expenditure and joint kinematics of a patient with an internal hemipelvectomy who underwent a wide excision of a chondrosarcoma followed by intensive rehabilitation.

Outcomes: Walking speed $(1.6 \mathrm{~m} / \mathrm{s})$, stride length $(1.7 \mathrm{~m})$, stride width $(0.13 \mathrm{~m})$ and good symmetry in step length (right $0.85 \mathrm{~m}$ and left data $0.80 \mathrm{~m}$ ) and stance time (right $62 \%$ and left $59 \%$ ) indicate a walking pattern similar to the asymptomatic able bodied. Oxygen consumption was $16.6 \mathrm{ml} / \mathrm{kg} / \mathrm{min}$ and oxygen cost $0.17 \mathrm{ml} / \mathrm{kg} / \mathrm{m}$.

Discussion: Following the intensive 2-year rehabilitation program this patient's gait pattern was similar to a control group with no statistically significant difference in the movement of the left and right knee and ankle joints. Some differences were identified against the control group in respect of the movement of the pelvis and hips, but this is partly to be expected. This case study highlights the functional outcomes that can be achieved following limb salvage surgery with intensive rehabilitation and a high level of motivation from the individual.
\end{abstract}

\section{$>$ IMPLICATIONS FOR REHABILITATION}

- Advancements in chemotherapy, imaging and surgical techniques have allowed more patients to be treated with internal hemipelvectomy.

- The rehabilitation outcomes following an internal hemipelvectomy have not been well identified and there is little gait analysis data.

- This case study highlights the functional outcomes that can be achieved following limb salvage surgery with intensive rehabilitation and motivation from the individual.

- Rehabilitation following internal hemipelvectomy should include goal setting, lymphoedema management, physiotherapy and an exercise program.

\section{Introduction}

Malignant bone tumors are rare neoplasms with an incidence of around one to two new cases per 100,000 individuals per year [1]. Chondrosarcoma is a malignant tumor, consisting of cartilaginous tissue without osteoid and accounts for approximately $20 \%$ of bone sarcomas [2]. On average, 113 new cases of chondrosarcoma were registered each year between 1979 and 2007 and are marginally more common in men with a male to female ratio of 1.21:1. The most common skeletal sites are the bones of the pelvis with the ilium being the most frequently involved bone [3]. The five-year survival is $89 \%$ for patients with grade 1 and the combined group of patients with grade 2 and 3 have a five-year survival of $53 \%$ [3].
Pelvic tumors are difficult and challenging to treat due to the complexity of the bony anatomy and the proximity to the bladder, rectum and neurovascular structures [4]. Pelvic tumors have until recently been treated with standard hemipelvectomy, that is, hindquarter amputation [5]. However, advancements in chemotherapy, radiation therapy, imaging and surgical techniques allow more patients to be treated with internal hemipelvectomy therefore salvaging the limb. This has led to better survival rates, quality of life and the opportunity for patients to obtain better postoperative mobility compared to standard hemipelvectomy treatment [6].

The extent of the surgery is dependent on tumor location, size and margins and invasiveness with involvement of vital neurovascular structures [7]. Internal hemipelvectomy is now considered 
the surgical procedure of choice for local control of malignant bone tumors in more than $90 \%$ of patients [8]. It is a surgical procedure of partial to complete unilateral resection of bone and soft tissue of the pelvis with preservation of the ipsilateral leg. It is indicated when tumor resection with wide margins can be obtained without sacrificing the remaining neurovascular tissue [9].

An extensive literature search found limited published data on rehabilitation and outcomes, such as gait parameters, following internal hemipelvectomy in limb salvage patients. The majority of studies focused on prosthetic use and basic functional outcome measures. Houdek [10] found that the walking speed of hemipelvectomy patients using a prosthesis was slower compared to those using crutches; Akiyama [11] found hemipelvectomy patients' gait pattern was improved by the use of a hip stabilizer; and Benedetti [12] concluded that walking speed and stride length were reduced in all patients with prosthetic reconstruction compared to a control group.

The aim of this case study is to describe temporal and spatial parameters, metabolic energy expenditure and joint kinematics of an internal hemipelvectomy patient who underwent a wide excision of a chondrosarcoma followed by intensive rehabilitation.

The individual in this case study has provided informed, verbal and written consent for this paper to be submitted for publication.

\section{Case description}

The patient is a 53-year-old male (height: $174 \mathrm{~cm}$, weight: $75 \mathrm{~kg}$ ) in the UK Armed Forces. Prior to surgery, he was fit and active and enjoyed a variety of outdoor sports such as water skiing, sailing, skiing, cycling and swimming. His past medical history included the diagnosis of an osteosarcoma of the left side of the pelvis at the age of 28 , following a 2-year history of left hip pain. He underwent surgery to remove the osteosarcoma and a donor bone graft in 1989. No lymph nodes were involved at this time and there were no post-operative problems.

In 2012, a chondrosarcoma around the left acetabulum was identified on MRI following a 6-year history of worsening hip pain. The tumor was classified as a grade 2 according to the musculoskeletal tumor society staging system [13]. In March 2013, he underwent a wide local excision of the chondrosarcoma involving a complex type-2 hemipelvectomy pelvic resection with lymph node clearance which involved resection of the acetabulum alone and is termed a peri-acetabular resection. The left hip joint was reconstructed which incorporated a pediculed cup inserted in the position of the hip joint which was reinforced with three trabecular buttresses and cement within the joint to provide good fixation and a stable hip joint construct. A Zimmer ${ }^{\circledR} M / L$ taper was inserted into the femur to complete the total hip replacement. The pelvic resection was extensive including the removal of the left ischial tuberosity and symphysis pubis as identified on Figure 1 and Figure 2. He did not receive any radiotherapy or chemotherapy.

The patient was admitted to the Defense Medical Rehabilitation Centre (DMRC), Headley Court for intensive inpatient rehabilitation, which is a Ministry of Defense in-patient based rehabilitation facility. The Complex Trauma department is a multidisciplinary team involving Physiotherapists, Exercise Rehabilitation Instructors, Occupational Therapists, Nurses, Social Workers, Medical Team, Podiatrists, Psychologists and a Rehabilitation Assistant. Patients have varying lengths of admission depending on their individual needs and typically go back

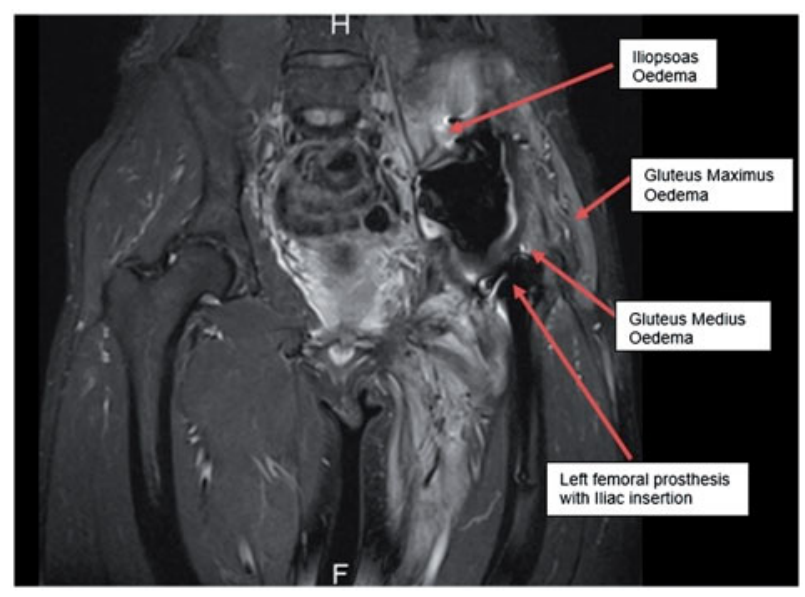

Figure 1. Coronal fluid sensitive fat saturated MRI of the pelvis showing disruption and edema of the adductors with insertion onto the anterior margin of pubic symphysis and rectus abdominis.

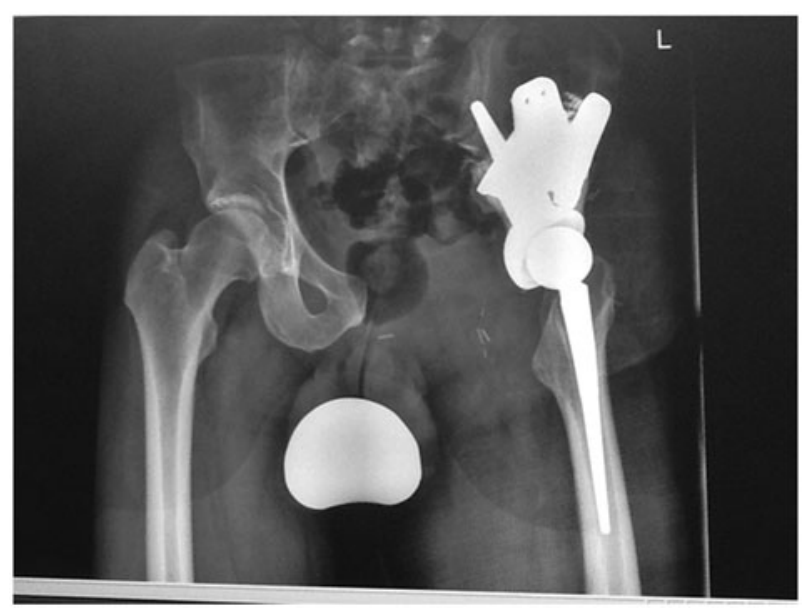

Figure 2. X-ray of patient's pelvis showing an Internal hemipelvectomy with limb sparing. Reconstruction with pedicled cup inserted in the position of the hip and trabecular metal implant.

home for a period of recovery leave in between each admission. This patient had 13 separate admissions between 3 and 6 weeks in duration over a two-year period. Goals were set at the start of every admission and reviewed throughout. An individual weekly timetable of sessions were provided at the start of each week and included hydrotherapy, yoga, circuit training, swimming and time to complete his individual exercise program. Due to the clearance of lymph nodes, lymphoedema management became a vital part of rehabilitation and included the use of compression hosiery, a Pulse Press Intermittent Pneumatic Compression Machine with a whole leg boot plus lower limb elevation. He received approximately an hour of individual Physiotherapy treatment per day as well as scheduled time with other healthcare professionals. The patient's long-term goal was to mobilize independently and return to cycling.

\section{Intervention}

Gait analysis took place within the gait laboratory at DMRC, Headley Court and was part of an in-house clinical gait analysis service. Major joint segments were identified using lower limb retro-reflective markers and these were attached onto the skin using the protocol described in Jarvis et al. [14]. The landmarks were determined by palpation and the reflective markers were 
Table 1. Temporal and spatial parameters, metabolic energy expenditure and functional outcomes.

\begin{tabular}{|c|c|c|c|}
\hline & \multicolumn{2}{|c|}{ Patient (2015) } & Control data \\
\hline Walking speed & \multicolumn{2}{|c|}{$1.6 \mathrm{~m} / \mathrm{s}$} & $1.29 \mathrm{~m} / \mathrm{s}$ \\
\hline Stride length & \multicolumn{2}{|l|}{$1.7 \mathrm{~m}$} & $1.47 \mathrm{~m}$ \\
\hline Stride width & $\begin{array}{l}0.13 \mathrm{~m} \\
\text { Left }\end{array}$ & Right & $0.10 \mathrm{~m}$ \\
\hline Step length & $0.85 \mathrm{~m}$ & $0.80 \mathrm{~m}$ & $0.74 \mathrm{~m}$ \\
\hline Stance time (\% of gait cycle) & $59 \%$ & $62 \%$ & $63 \%$ \\
\hline Average pelvic tilt & $19.46^{\circ}$ & $19.46^{\circ}$ & \\
\hline Peak hip extension & $6.06^{\circ}$ & $3.46^{\circ}$ & \\
\hline Maximum hip adduction & $12.26^{\circ}$ & $10.20^{\circ}$ & \\
\hline Average hip rotation & $16.09^{\circ}$ & $16.17^{\circ}$ & \\
\hline Peak knee flexion during $25 \%$ of gait cycle & $25.96^{\circ}$ & $37.65^{\circ}$ & \\
\hline Peak knee flexion during gait cycle & $65.70^{\circ}$ & $71.26^{\circ}$ & \\
\hline \multicolumn{4}{|c|}{ Maximum dorsi-flexion during stance at ankle joint } \\
\hline \multicolumn{3}{|l|}{ Oxygen consumption (ml/kg/min) } & 11.34 \\
\hline Oxygen cost $(\mathrm{ml} / \mathrm{kg} / \mathrm{m})$ & \multicolumn{2}{|c|}{0.17} & 0.15 \\
\hline 6-Minute walk test (6MWT) & \multicolumn{2}{|c|}{$610 \mathrm{~m}$} & \\
\hline
\end{tabular}

Control data from Jarvis et al. [15].

positioned to track the movement of the pelvis, trunk, thigh and foot segments during gait. Kinematic data were collected at $120 \mathrm{~Hz}$ and ground reaction forces at $1200 \mathrm{~Hz}$. A static standing trial was recorded for the subject to calculate the location of joint centers. The subject mobilized independently while wearing trainers for a period of $5 \mathrm{~min}$.

A variety of functional and psychological outcome measures were used at the start of each admission to monitor progress. These included two screening and diagnostic tools for mental health disorders, the General Anxiety Disorder (GAD 7) and Patient Health Questionnaire (PHQ 9). Reintegration to Normal Living (RNLI) was used as it assesses global function in individuals after incapacitating illness or severe trauma and is based on 11 declarative statements. The 6 Minute Walk Test (6MWT) was a functional outcome measure and assesses distance walked over $6 \mathrm{~min}$.

A control group described in previous research $[14,15]$ was used to compare the results from this case study to. The control group were similarly matched all male military personnel (age 30 years $(S D=6)$ years, weight $78 \mathrm{~kg}(S D=8)$ and height $184 \mathrm{~cm}$ $(S D=0.07))$. Exactly the same data collection procedures for collection of temporal and spatial parameters, metabolic energy expenditure and joint kinematics were used for the control group and this case study.

\section{Results}

Temporal and spatial parameters, joint kinematics, oxygen consumption and functional outcomes are outlined in Table 1. All comparisons to a control group are from data published elsewhere $[14,15]$ using the same data collection methods as described here.

Step length is $0.80 \mathrm{~m}$ on the right and $0.85 \mathrm{~m}$ on the left. Right stance time is $62 \%$ and the left is $59 \%$. Stride length for the patient is $1.7 \mathrm{~m}$ compared to the control group of $1.47 \mathrm{~m}$ and walking speed is $1.6 \mathrm{~m} / \mathrm{s}$ for the patient compared to $1.29 \mathrm{~m} / \mathrm{s}$ in the control group. Oxygen consumption is $16.6 \mathrm{ml} / \mathrm{kg} / \mathrm{min}$ and oxygen cost are $0.17 \mathrm{ml} / \mathrm{kg} / \mathrm{min}$.

Figure 3 highlights the joint kinematics of the pelvis, hip, knee and ankle of the right and left legs during walking. On the left leg, compared to the control there is increased anterior pelvic tilt (point a) during left mid stance and increased left internal pelvic rotation throughout the gait cycle. The left average pelvic tilt is $19.46^{\circ}$. There is increased left hip flexion at the start of the gait cycle and a limitation into extension (point b), with an average of $6.06^{\circ}$ during stance phase. Left hip adduction (point c) reduces during swing through with the left hip remaining in external rotation throughout the gait cycle (point d). Movement patterns of the knees and ankles are similar to the control data (point e and $f$ ).

RNLI in July 2015 was $95.5 \%$ with a total score of $105 / 110$. On discharge, his GAD7 and PHQ9 scores were both 0. He achieved $614 \mathrm{~m}$ on the $6 \mathrm{MWT}$.

\section{Discussion}

Few studies have examined in detail the gait pattern and functional outcomes of patients following an internal hemipelvectomy [11] and functional outcome has seldom been evaluated with laboratory-based computer assisted gait analysis [12]. However, the high level of functional outcomes achieved and the gait analysis findings following this complex surgery prompted this case report.

The patient in this case report walked at a similar speed, step length and stride width to control data published elsewhere [15]. This is also considerably better than a study by Akiyama [11] which reported that following resection of tumors located around the pelvis the average walking speed of seven subjects in that study was $0.75 \mathrm{~m} / \mathrm{s}$. Similarly, Benedetti [12] described that in two groups of 10 patients each with different prosthetic reconstructions of the proximal femur. They found walking speed for these two groups to be $1.00 \mathrm{~m} / \mathrm{s}$ and $1.11 \mathrm{~m} / \mathrm{s}$. However, there are notable deviations in joint kinematics of the walking pattern of this patient compared to what is expected of symptom free gait patterns. There is limited left hip adduction during swing through, which could be associated with edema of the adductors and their insertion onto the anterior margin of pubic symphysis and rectus abdominis. Gluteus medius is absent and this may account for the left hip remaining in external rotation throughout the gait cycle. There is clumping of the common hamstring origin onto adductor magnus.

A study by Akiyama [11] assessed gait parameters following the resection of tumors located around the pelvis and found the average walking speed of the seven subjects was $0.75 \mathrm{~m} / \mathrm{s}$. Benedetti [12] used gait analysis to assess walking ability in two groups of 10 patients each with different prosthetic reconstructions of the proximal femur. They found walking speed for these two groups to be $1.00 \mathrm{~m} / \mathrm{s}$ and $1.11 \mathrm{~m} / \mathrm{s}$. In comparison, this patient walked at a speed of $1.6 \mathrm{~m} / \mathrm{s}$. Chetta et al. [16] reported a mean 6-min walk distance a $614 \mathrm{~m} \pm 56 \mathrm{~m}$ for 102 healthy subjects (54 females and 48 males) between the ages of 20 and 50 years. The patient within this case report mobilized $610 \mathrm{~m}$ at the end of his rehabilitation period, which is therefore comparable to the distance completed by the healthy subjects in the aforementioned survey.

Although this patient reported mild symptoms of depression in 2013 the subject left the rehabilitation pathway reporting no symptoms of depression of generalized anxiety disorder in 2015. The functional and mental health outcomes at discharge are comparable to the general population norms optimizing the prospect of full integration back into society. A study comparing the patient reported outcome measure (PROM) reintegration to normal living index (RNLI) outcomes in amputees versus limb salvage patients with lower extremity sarcoma [17] found that those in the amputation group scored lower (84.4) compared to those in the limb sparing group (97, out of 110). The concept of RNLI is closely allied to functional performance [18] which is supported in this case study as the patient went from scoring $75.3(68.5 \%)$ to 105 out of $110(95.5 \%)$. This demonstrates that his perception of 
Pelvic Tilt
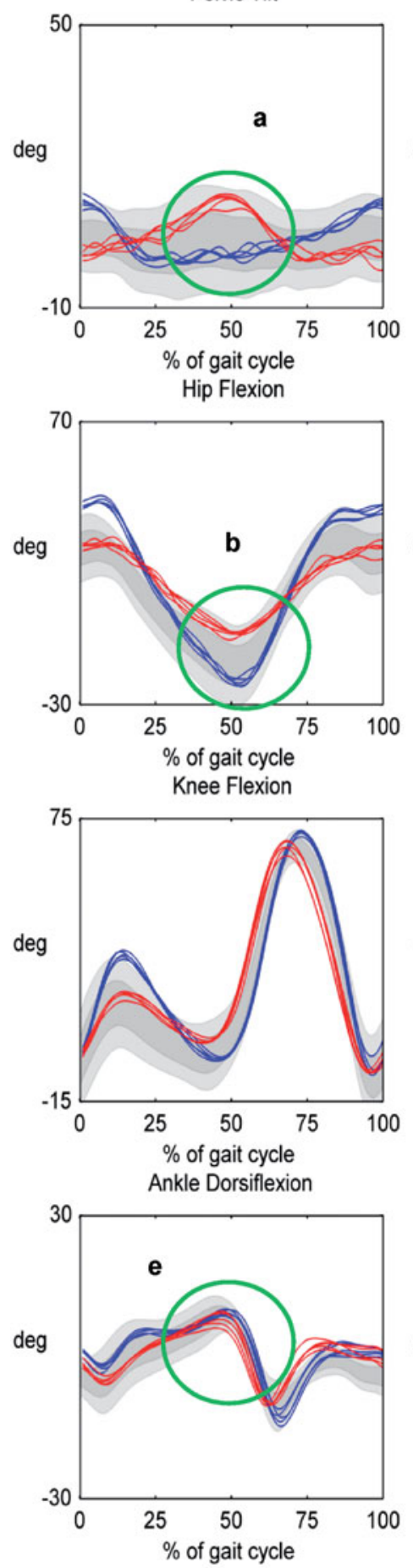

Pelvic Obliquity
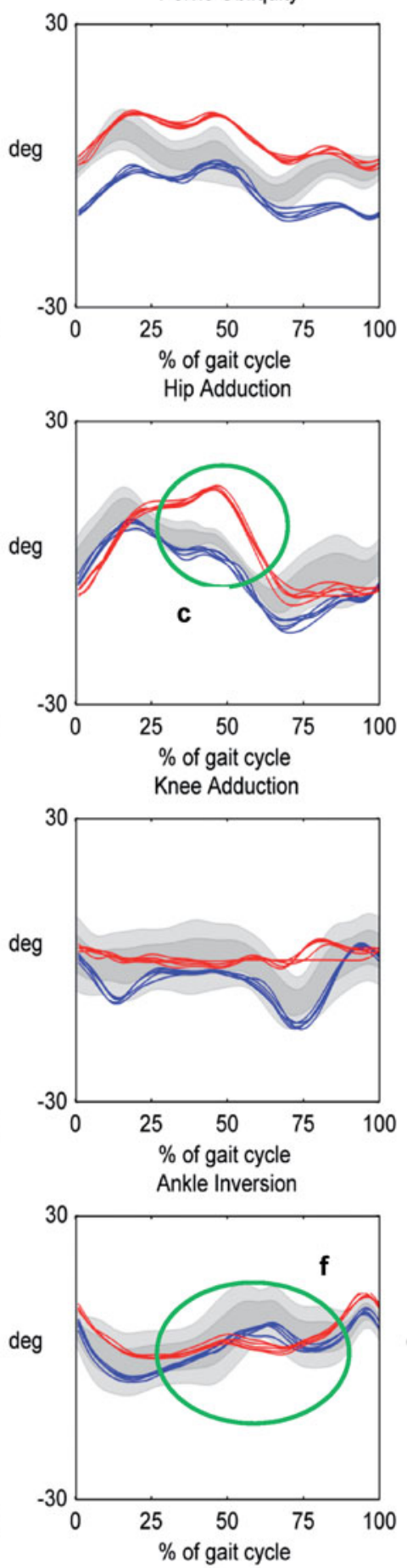

Pelvic Rotation
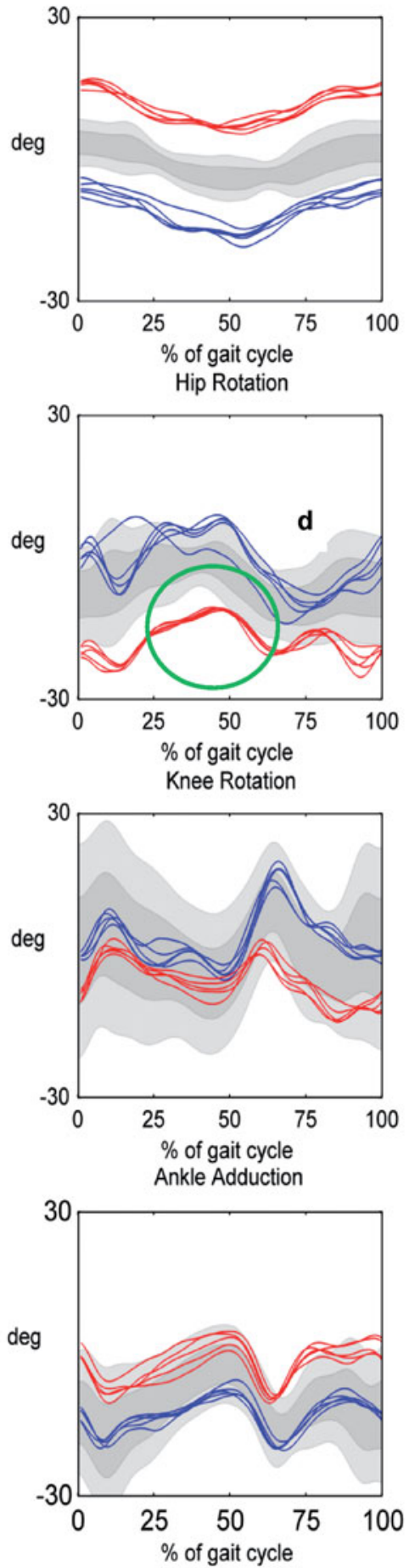

Figure 3. Joint kinematics of the pelvis, hip, knee and ankle of the left (red) and right (blue) legs during walking. Grey shaded area represents control sample.

his capabilities and objective indicators of physical, social and psychological performance were markedly improved by the time he discharged.

This is the first case study that the author is aware of that presents gait data in a patient who has undergone a hemipelvectomy. It is recognized that the surgery is individual and the results are specific to this particular patient who benefitted from the rehabilitation facilities provided by the Ministry of Defense. Therefore, the functional outcomes and gait analysis data may be difficult to replicate to a wider population without sufficient time and investment. However, the general principles of rehabilitation are considered to be applicable for anyone and illustrative of what can be accomplished.

\section{Conclusions}

The results set out in this case study highlight the functional outcomes that can be achieved following a hemipelvectomy with regular intensive rehabilitation and a high level of motivation from the individual. Following an intensive 2-year rehabilitation program, this patient demonstrated a gait pattern that was very similar to a control group of asymptomatic, able bodied military males. 
The patient now swims, cycles and attends the gym regularly and has returned to full time employment.

\section{Acknowledgements}

We would like to acknowledge Colonel Alan Mistlin and Dr Edward Sullon for their help with the clinical interpretation of the X-ray and MRI.

\section{Disclosure statement}

The authors report no declarations of interest.

\section{References}

[1] Rozeman L, Cleton-Jansen A, Hogendoom P. Pathology of primary malignant bone and cartilage tumours. International Orthopaedics (Sicot). 2006;30:437-444.

[2] Gitelis S, Bertoni F, Picci P, et al. Chondrosarcoma of the bone. The experience at the Instituto Orthopedico Rizzoli. J Bone Joint Surg Am, 1981;63:1248-1257.

[3] Fletcher C, Unni K, Mertens F., editors. World Health Organization classification of tumours. Pathology and genetics of tumours of soft tissue and bone. Lyon: International Agency for Research on Cancer (IARC); 2002.

[4] Shao QD, Yan X, Sun JY, et al. Internal hemipelvectomy with reconstruction for primary pelvic neoplasm: a systematic review. ANZ J Surg. 2014;85:553-560.

[5] Asavamongkolkul A, Waikakul S. Using Polypropylene mesh graft for soft tissue reconstruction in internal hemipelvectomy; a case report. World J Surg Onc. 2012;10:124.

[6] Wirbel R, Schulte M, Maier B, et al. Chondrosarcoma of the pelvis: oncologic and functional outcome. Sarcoma. 2000;4:161-168.

[7] Beck $L$, Einertson $M$, Winemiller $M$, et al. Functional outcomes and quality of life after tumor-related hemipelvectomy. Phys Ther. 2008;88:916-927.

[8] Gosheger G, Gebert C, Ahrens H, et al. Endoprosthetic reconstruction in 250 patients with sarcoma. Clin Orthopaed Related Res. 2006;450:164-171.
[9] Guder W, Hardes J, Gosheger G, et al. Analysis of surgical and oncological outcome in internal and external hemipelvectomy in 34 patients above the age of 65 years at a mean follow up of 56 months. Bmc Musculoskelet Disord. 2015;16:33.

[10] Houdek M, Andrews $K$, Kralovec $M$, et al. Functional outcome measures of patients following hemipelvectomy. Prosthet Orthot Int. 2016;40:566-572.

[11] Akiyama T, Saita K, Ogura K, et al. The effect of an external hip joint stabiliser on gait function after surgery for tumours located around the circumference of the pelvis: analysis of seven cases of internal hemipelvectomy or proximal femur resection. Int Orthop. 2016; 40:561-567.

[12] Benedetti M, Bonatti E, Malfitano C, et al. Comparison of allograft-prosthetic composite reconstruction and modular prosthetic replacement in proximal femur bone tumors. Functional assessment by gait analysis in 20 patients. Acta Orthopaedica. 2013;84:218-223.

[13] Enneking W, Spanier S, Goodman M. A system for the surgical staging of musculoskeletal sarcoma. Clin Orthopaed Related Res. 1980;153:106-120.

[14] Jarvis H, Baker R, Bennett A, Twiste M, Phillip R. Kinematics, kinetics and gait profile score in highly functional amputees. Prosthet Orthot Int. 2015;39:258.

[15] Jarvis $H$, Bennett $A$, Twiste $M$, et al. Temporal spatial and metabolic measures of walking in highly functional individuals with lower limb amputations. Arch Phys Med Rehabil. 2017;98:1389-1399.

[16] Chetta A, Zanini A, Pisi G, et al. Reference values for the 6 minute walk test in healthy subjects 20-50 year olds. Resp Med. 2006;100:1573-1578.

[17] Davis A, Devlin M, Griffin A, et al. Functional outcome in amputation versus limb sparing of patients with lower extremity sarcoma: a matched case control study. Arch Phys Med Rehabil. 1999;80:615-618.

[18] Wood-Dauphinee S, Williams J. Reintegration to normal living as a proxy to quality of life. J Chronic Dis. 1987; 40:491-502. 